\title{
Chemical Evolution of a Protoplanetary Disk
}

\author{
Dmitry A. Semenov ${ }^{1}$ \\ ${ }^{1}$ Max Planck Institute for Astronomy, Königstuhl 17, D-69117 Heidelberg, Germany \\ email: semenov@mpia.de
}

\begin{abstract}
In this paper we review recent progress in our understanding of the chemical evolution of protoplanetary disks. Current observational constraints and theoretical modeling on the chemical composition of gas and dust in these systems are presented. Strong variations of temperature, density, high-energy radiation intensities in these disks, both radially and vertically, result in a peculiar disk chemical structure, where a variety of processes are active. In hot, dilute and heavily irradiated atmosphere only the most photostable simple radicals and atoms and atomic ions exist, formed by gas-phase processes. Beneath the atmosphere a partly UVshielded, warm molecular layer is located, where high-energy radiation drives rich ion-molecule and radical-radical chemistry, both in the gas phase and on dust surfaces. In a cold, dense, dark disk midplane many molecules are frozen out, forming thick icy mantles where surface chemistry is active and where complex polyatomic (organic) species are synthesized. Dynamical processes affect disk chemical composition by enriching it in abundances of complex species produced via slow surface processes, which will become detectable with ALMA.
\end{abstract}

Keywords. accretion disks, astrochemistry, diffusion, line: formation, molecular processes, protoplanetary disks, submillimeter, techniques: interferometric, turbulence

\section{Introduction}

Protoplanetary disks (PPDs) are ubiquitous around young stars (e.g., Lin \& Papaloizou 1980; Lissauer 1987). Their chemical composition and physical properties regulate the efficiency and timescale of planet formation. Molecules and dust serve as heating and cooling agents of the gas, while dust grains also dominate the disk opacities. Molecular lines observed at infrared and (sub-)millimeter wavelengths are useful probes of physical conditions (temperature, density, kinematics, turbulence) in PPDs.

Over the past decade significant progress has been achieved in our understanding of disk chemical composition, both theoretically and observationally. Since molecular hydrogen is not observable when it is cold, we have to rely on other trace species to infer disk physics and chemistry (Table 1). Multi-molecule, multi-transition interferometric observations, coupled to line radiative transfer and chemical modeling, allowed to constrain disk sizes, kinematics, distribution of temperature, surface density, and molecular column densities (see reviews by Bergin et al. (2007) and Dutrey et al. (2007a)).

Observations of thermal dust emission are employed to constrain dust properties, infer disk masses, whereas IR spectroscopy is used to study mineralogical composition of dust in PPDs. Recently, with space-borne (Spitzer) and ground-based (Keck, VLT, Subaru) infrared telescopes, molecules have been detected in very inner zones of planet-forming systems, at $r \lesssim 1-10$ AU.

The conditions of planets formation in the early Solar system have been revealed by a detailed analysis of chemical and mineralogical composition of meteoritic samples and cometary dust particles (e.g., Bradley 2005). The recent Stardust and Genesis space missions have returned first samples of pristine materials, likely of cometary origin, showing a complex structure of high-temperature crystalline silicates embedded in low-temperature 
Table 1. Molecular species commonly utilized to study disks

\begin{tabular}{llcccc}
\hline Tracer & Quantity & Midplane & $\begin{array}{c}\text { Molecular } \\
\text { layer }\end{array}$ & Atmosphere & $\begin{array}{c}\text { Inner } \\
\text { zone }\end{array}$ \\
\hline${ }^{12} \mathrm{CO},{ }^{13} \mathrm{CO}$ & Temperature & $\mathrm{mm}^{*}$ & $\mathrm{~mm}$ & $\mathrm{~mm}$ & $\mathrm{IR}$ \\
$\mathrm{H}_{2}$ & - & 0 & 0 & 0 & $\mathrm{IR}$ \\
$\mathrm{NH}_{3}$ & - & $\mathrm{cm}$ & $\mathrm{cm}$ & 0 & 0 \\
$\mathrm{CS}, \mathrm{H}_{2} \mathrm{CO}$ & Density & 0 & $\mathrm{~mm}$ & 0 & $\mathrm{IR}$ \\
$\mathrm{CCH}, \mathrm{HCN}, \mathrm{CN}$ & Photochemistry & 0 & $\mathrm{~mm}$ & 0 & $\mathrm{IR}$ \\
$\mathrm{HCO}^{+}$ & Ionization & 0 & $\mathrm{~mm}$ & 0 & 0 \\
$\mathrm{~N}_{2} \mathrm{H}^{+}$ & - & $\mathrm{mm}$ & 0 & 0 & 0 \\
$\mathrm{C}^{+}$ & - & 0 & 0 & $\mathrm{IR}$ & $\mathrm{IR}$ \\
complex organics & Surface & $\mathrm{IR}^{* *}$ & $\mathrm{IR}-\mathrm{cm}$ & 0 & $\mathrm{IR}, \mathrm{mm}$ \\
$\mathrm{DCO}^{+}, \mathrm{DCN}$, & processes & & & & \\
$\mathrm{H}_{2} \mathrm{D}^{+}$ & Deuterium & $\mathrm{mm}$ & $\mathrm{mm}$ & 0 & 0 \\
\hline
\end{tabular}

* - "mm/cm" and "IR" mean radio-interferometric and infrared observations, respectively.

** - Complex molecules frozen onto dust surfaces could be detected through absorption lines in infrared, while the gas-phase counterparts emit at (sub-) millimeter frequencies.

condensates (Brownlee et al. 2004; Flynn et al. 2006; Brownlee et al. 2008). The presence of crystalline silicates in outer regions of protoplanetary disks has also been revealed (e.g., van Boekel et al. 2004; Juhász et al. 2010). An isotopic analysis of refractory condensates in unaltered chondritic meteorites shows strong evidence that the inner part of the Solar Nebula has been almost completely mixed during the first several Myr of evolution (e.g., Boss 2004; Ciesla 2009).

These intriguing findings are partly understood in modern astrochemical models of protoplanetary disks (Aikawa \& Herbst 1999; Markwick et al. 2002; van Zadelhoff et al. 2003; Tscharnuter \& Gail 2007; Agúndez et al. 2008; Woods \& Willacy 2009; Visser et al. 2009b; Walsh et al. 2010; Semenov \& Wiebe 2011). The major result of the chemical modeling is that disks have a layered chemical structure due to heavy freeze-out of gas-phase molecules in the cold midplane and their photodissociation in the atmosphere. Observed column densities of $\mathrm{CO}, \mathrm{HCO}^{+}, \mathrm{N}_{2} \mathrm{H}^{+}, \mathrm{CN}, \mathrm{HCN}, \mathrm{HNC}$, CS, etc. are qualitatively agree with the chemical models.

In this paper we briefly review the major observational findings and modeling predictions for the chemical composition and evolution of protoplanetary disks.

\section{Observational constrains on composition of gas and ices}

Apart from $\mathrm{CO}$ and its isotopologues, and occasionally $\mathrm{HCO}^{+}, \mathrm{DCO}^{+}, \mathrm{CN}, \mathrm{HCN}$, DCN, $\mathrm{CCH}, \mathrm{H}_{2} \mathrm{CO}$, and $\mathrm{CS}$, the molecular content of protoplanetary disks remains largely unknown (e.g., Dutrey et al. 1997; Kastner et al. 1997; Aikawa et al. 2003; Thi et al. 2004; Piétu et al. 2007; Qi et al. 2008; Henning et al. 2010). Molecular line data are limited in sensitivity and resolution. Thus the spatial distribution of molecular abundances is still poorly determined (e.g., Piétu et al. 2005; Dutrey et al. 2007b; Panić et al. 2009). Observational facilities such as the Plateau de Bure interferometer (France) and Submillimeter Array (USA) have permitted measurements of several brightest nearby disks (DM Tau, LkCa 15, AB Aur, and TW Hya). Soon, with the Atacama Large Millimeter Array entering it's full power in 2013, we will make substantial progress in our knowledge of molecular disk structure.

Typically, studies of disk physics begin with observations of bright CO lines. These lines are thermally excited at densities $\sim 10^{3}-10^{4} \mathrm{~cm}^{-3}$. The ${ }^{12} \mathrm{CO}$ lines are optically thick and 
their intensities measure kinetic temperature in the upper disk layer (e.g., Dutrey et al. 1997). The lines of less abundant ${ }^{13} \mathrm{CO}$ and $\mathrm{C}^{18} \mathrm{O}$ are typically optically thin or partially optically thick and are sensitive to both temperature and corresponding column densities throughout the entire disk. Strong CO lines are most suitable for accurate determination of disk kinematics as well as orientation and geometry. Their measured widths indicate that turbulence in disks is subsonic, with typical velocities of about $0.05-0.2 \mathrm{~km} \mathrm{~s}^{-1}$ (Dutrey et al. 2007a; Semenov et al. 2010a; Hughes et al. 2011).

It has been found that disks appear progressively larger from observations of dust continuum, onwards to $\mathrm{C}^{18} \mathrm{O},{ }^{13} \mathrm{CO}$, and ${ }^{12} \mathrm{CO}$, respectively, with typical values of $300-1000$ AU. This is a manifestation of selective isotopic photodissociation. Most disks exhibit a vertical temperature gradient, ranging from $\sim 10 \mathrm{~K}$ at the midplane to $\sim 50-100 \mathrm{~K}$ in the atmosphere region, as determined by physical models (e.g., Dartois et al. 2003; Qi et al. 2006; Piétu et al. 2007; Isella et al. 2010), though several disks with large inner cavities do not show evidence for such a gradient (e.g., GM Aur and LkCa 15; Dutrey et al. 2008; Hughes et al. 2009).

By comparing intensities of 6-5 to 2-1 CO transitions it was found by Qi et al. (2006) that the TW Hya disk has a surface region that is superheated, so that an additional heating mechanism is required, possibly stellar X-ray radiation (Glassgold et al. 1997). A significant reservoir of very cold $\mathrm{CO}, \mathrm{HCO}^{+}, \mathrm{CN}$ and $\mathrm{HCN}$ gases has been found in the disk of DM Tau at temperatures $\lesssim 6-17 \mathrm{~K}$, which cannot be explained by conventional chemical models without invoking a non-thermal desorption or transport mechanism (e.g., Semenov et al. 2006; Aikawa 2007; Hersant et al. 2009).

The second most readily observed molecular species in disks is $\mathrm{HCO}^{+}$. The low-lying transitions of this ion are thermalized at densities of about $10^{5} \mathrm{~cm}^{-3}$. This is one of the most abundant charged molecule in disks, the other being $\mathrm{C}^{+}$(not observable at millimeter wavelengths) and $\mathrm{H}_{3}^{+}$(lacking dipole moment). The ionization degree measured from $\mathrm{HCO}^{+}$is $\sim 10^{-10}-10^{-9}$ inside warm molecular disk layer (Qi et al. 2003; Dutrey et al. 2007b).

Less strong lines of $\mathrm{C}_{2} \mathrm{H}, \mathrm{CN}$, and $\mathrm{HCN}$ are sensitive to the intensity and shape of the incident UV spectrum and are excellent tracers of photochemistry (e.g., Bergin et al. 2003a). Recently detected $\mathrm{DCO}^{+}$and $\mathrm{DCN}$ have abundances that are about $1-10 \%$ of the $\mathrm{HCO}^{+}$and $\mathrm{HCN}$ densities (Qi et al. 2008). It remains to be verified whether such a large degree of deuteration is a heritage of cloud chemistry or produced in situ.

A key observational result is that molecular abundances are depleted by factors 5100 compared to the values in the Taurus Molecular Cloud (Dutrey et al. 2007a). Since disks have higher densities up to $10^{7}-10^{10} \mathrm{~cm}^{-3}$ and enshrouded in stronger ionizing radiation fields, this depletion can be attributed to a combined effect of photodissociation and freeze-out.

The results from the Infrared Space Observatory and Spitzer telescope have proven the existence of a significant amount of frozen material and various types of silicates and polycyclic aromatic hydrocarbons (PAH) in disks (van den Ancker et al. 2000; van Dishoeck 2004; Bouwman et al. 2008). The PAH features at $\sim 3-12 \mu \mathrm{m}$ probe the incident radiation field and density distribution of the upper disk (e.g., Habart et al. 2004). Recently, with space-borne (Spitzer) and ground-based (Keck, VLT, Subaru) infrared telescopes, molecules have been detected in very inner zones of planet-forming systems, at $r \lesssim 1-10$ AU. Rotational-vibrational emission lines from $\mathrm{CO}, \mathrm{CO}_{2}, \mathrm{C}_{2} \mathrm{H}_{2}, \mathrm{HCN}, \mathrm{OH}$, $\mathrm{H}_{2} \mathrm{O}$ imply a rich chemistry driven by endothermic reactions or reactions with activation barriers and photoprocesses (Lahuis et al. 2006; Carr \& Najita 2008; Salyk et al. 2008; Pontoppidan et al. 2008; Pascucci et al. 2009; van der Plas et al. 2009; Salyk et al. 2011). Through $I S O$ and Spitzer infrared spectroscopy abundant ices in cold disk regions 


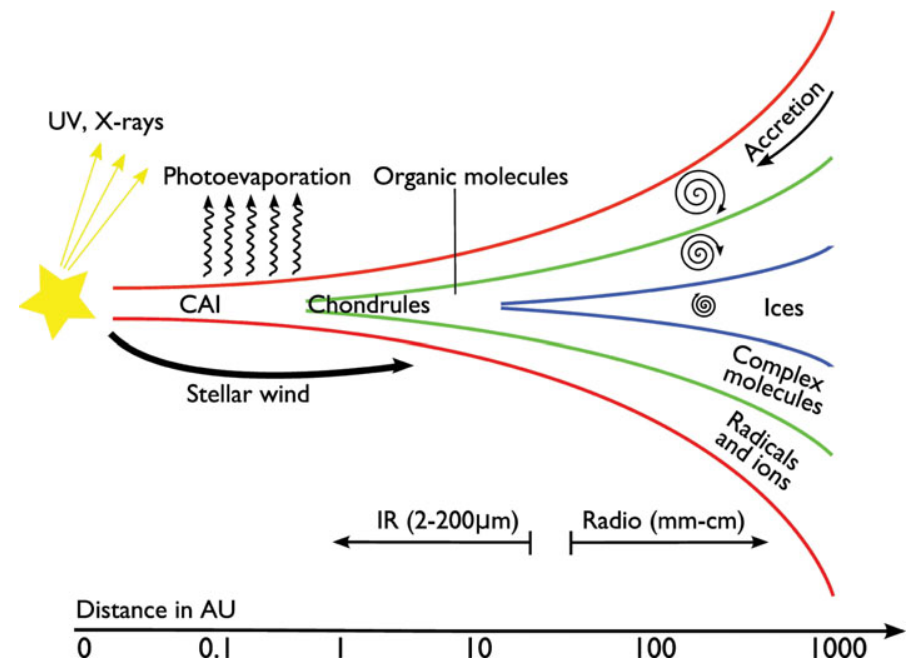

Figure 1. Physical and chemical structure of a protoplanetary disk. Timescales are rough estimates and given for a radius of about $100 \mathrm{AU}$.

consisting of water ice and substantial amounts $(\sim 1-30 \%)$ of volatile materials like $\mathrm{CO}, \mathrm{CO}_{2}, \mathrm{NH}_{3}, \mathrm{CH}_{4}, \mathrm{H}_{2} \mathrm{CO}$, and $\mathrm{HCOOH}$ have been detected (e.g., Pontoppidan et al. 2005; Terada et al. 2007; Zasowski et al. 2009).

In Table 1 the various molecules used to study protoplanetary disks are summarized.

\section{Global evolutionary picture of disk chemical structure}

The current scheme of the disk chemical structure is presented in Fig. 1. The disk can be divided into 4 distinct chemical regions, primarily determined by temperature. The warm "inner zone" corresponds to radii $<20$ AU that are accessible with the IR instruments, while the other 3 regions dubbed "midplane", "molecular layer", and "atmosphere" represent the outer disk regions $(r>20-50$ AU) that are observed with radio-interferometers.

Dense midplane is opaque to the UV and X-ray radiation and remains cold and essentially neutral $(10-20 \mathrm{~K})$. Chemical complexity in this region is initially reached by fast ion-molecular reactions in the gas, followed by slow accretion onto grains and surface reactions (mostly hydrogenation). Once formed, a molecule is seldom re-emitted into the gas phase. Typical chemical timescales for this region are determined by freeze-out and surface reactions, and are $>10^{5}-10^{6}$ years in the outer disk. In the very inner part, the midplane is hot due to viscous accretion heating $(T \gtrsim 50 \mathrm{~K})$ and gas-grain interactions are not important, so the chemical timescale is due to gas-phase neutral-neutral reactions ( $\sim 100$ years). The planet-forming zone is in general in a chemical steady-state, which allows the application of simple condensation/evaporation thermodynamical chemical models.

Adjacent to the midplane lies a less dense, warmer layer $(T \sim 30-70 \mathrm{~K})$ that is partly shielded from stellar and interstellar UV/X- ray radiation. Young stars emit intense nonthermal UV and thermal and variable X-ray radiation fields that strongly mediate disk chemistry at intermediate heights (e.g., Bergin et al. 2007). UV excess in T Tauri stars is related to their chromospheric activity (e.g., Bouvier et al. 2007). Soft X-ray radiation 
Table 2. Chemical reactions active in disks

\begin{tabular}{|c|c|c|c|c|c|}
\hline Process & Formula & Midpl & lecu & osp & $\begin{array}{l}\text { Inner } \\
\text { zone }\end{array}$ \\
\hline $\begin{array}{l}\text { Bond formation } \\
\text { Radiative association } \\
\text { Surface formation } \\
\text { Three-body }\end{array}$ & $\begin{array}{l}\mathrm{A}+\mathrm{B} \rightarrow \mathrm{AB}+\mathrm{h} \nu \\
\mathrm{A}+\mathrm{B} \| \mathrm{gr} \rightarrow \mathrm{AB}+\mathrm{gr} \\
\mathrm{A}+\mathrm{B}+\mathrm{M} \rightarrow \mathrm{AB}+\mathrm{M}\end{array}$ & $\begin{array}{c}\mathrm{X} \\
\mathrm{X} \\
0\end{array}$ & $\begin{array}{c}\mathrm{X} \\
\mathrm{X} \\
0\end{array}$ & $\begin{array}{l}\mathrm{X} \\
0 \\
0\end{array}$ & $\begin{array}{l}\mathrm{X} \\
0 \\
\mathrm{X}\end{array}$ \\
\hline $\begin{array}{l}\text { Bond destruction } \\
\text { Photodissociation } \\
\text { Dissociation by CRP } \\
\text { Dissociation by X-rays } \\
\text { Dissociative } \\
\text { recombination }\end{array}$ & $\begin{array}{l}\mathrm{AB}+\mathrm{h} \nu \rightarrow \mathrm{A}+\mathrm{B} \\
\mathrm{AB}+\mathrm{CRP} \rightarrow \mathrm{A}+\mathrm{B} \\
-\mathrm{AB}^{+}+\mathrm{e}^{-} \rightarrow \mathrm{A}+\mathrm{B}\end{array}$ & $\begin{array}{l}0 \\
\mathrm{X} \\
0 \\
\mathrm{X}\end{array}$ & $\begin{array}{l}X \\
X \\
X \\
X\end{array}$ & $\begin{array}{l}\mathrm{X} \\
0 \\
\mathrm{X} \\
\mathrm{X}\end{array}$ & $\begin{array}{l}\mathrm{X} \\
0 \\
\mathrm{X} \\
\mathrm{X}\end{array}$ \\
\hline $\begin{array}{l}\text { Bond restructuring } \\
\text { Neutral-neutral } \\
\text { Ion-molecule } \\
\text { Charge transfer }\end{array}$ & $\begin{array}{l}\mathrm{A}+\mathrm{BC} \rightarrow \mathrm{AB}+\mathrm{C} \\
\mathrm{A}^{+}+\mathrm{BC} \rightarrow \mathrm{AB}^{+}+\mathrm{C} \\
\mathrm{A}^{+}+\mathrm{BC} \rightarrow \mathrm{A}+\mathrm{BC}^{+}\end{array}$ & $\begin{array}{l}X \\
X \\
X\end{array}$ & $\begin{array}{l}\mathrm{X} \\
\mathrm{X} \\
\mathrm{X}\end{array}$ & $\begin{array}{l}0 \\
\mathrm{X} \\
\mathrm{X}\end{array}$ & $\begin{array}{l}\mathrm{X} \\
\mathrm{X} \\
\mathrm{X}\end{array}$ \\
\hline $\begin{array}{l}\text { Unchanged bond } \\
\text { Photoionization } \\
\text { Ionization by CRP } \\
\text { Ionization by X-rays }\end{array}$ & $\begin{array}{l}\mathrm{AB}+\mathrm{h} \nu \rightarrow \mathrm{AB}^{+}+\mathrm{e}^{-} \\
\mathrm{AB}+\mathrm{CRP} \rightarrow \mathrm{AB}^{+}+\mathrm{e}^{-} \\
-\end{array}$ & $\begin{array}{l}0 \\
\mathrm{X} \\
0\end{array}$ & $\begin{array}{l}X \\
X \\
X\end{array}$ & $\begin{array}{c}\mathrm{X} \\
0 \\
\mathrm{X}\end{array}$ & $\begin{array}{c}\mathrm{X} \\
0 \\
\mathrm{X}\end{array}$ \\
\hline
\end{tabular}

has likely the same origin, while harder X-rays are produced in magnetic reconnection loops or due to jets (e.g., Güdel \& Nazé 2009).

These energetic photons dissociate and ionize gas and photodesorb surface species, thus enriching the gas composition and initiating a rich chemistry. Abundances of most molecules attain high concentrations in this zone, and numerous molecular lines are excited and observable emission produced. Chemistry does not reach a steady state in this region and a typical timescale, as determined by surface chemistry and gas-grain interactions, exceeds $10^{5}$ years.

Above the intermediate layer a hot, dilute, and heavily irradiated disk atmosphere exists $(T \gtrsim 100 \mathrm{~K})$. This is a molecularly-poor region, where only simple light hydrocarbons, their ions, and other radicals such as $\mathrm{CCH}$ and $\mathrm{CN}$ are able to survive. Chemical timescales are short ( $\sim 100$ years) and defined by photochemical processes and dissociate recombination.

\section{Gas-phase chemistry}

Models of chemical evolution employ a set of chemical reactions of relevance to conditions in disks. Modern astrochemical databases include up to 600 species involved in 4-7000 reactions (Smith et al. 2004; Le Petit et al. 2006; Woodall et al. 2007; Wakelam 2009 ). Only $10-20 \%$ of reaction rates have been studied in the laboratory or calculated theoretically and thus models are prone to inherent uncertainties (see, e.g., Wakelam et al. 2010). All reactions can be divided into 4 distinct groups that are dominant in different disk regions, see Table 2 (after van Dishoeck \& Black 1988).

Apart from the very dense inner zone, all reactions in disks are two-body processes. Three-body reactions become competitive only at $\lesssim 10$ AU, where $n \gtrsim 10^{10} \mathrm{~cm}^{-3}$ (Aikawa et al. 1999). The main processes leading to formation of molecular bonds are slow radiative association and surface reactions. Upon collision, a collisional complex 
in an excited state may form, which is stabilized with a low probability by emission of a photon (e.g., Bates 1951; Williams 1972; Herbst \& Klemperer 1973). For example, formation of light hydrocarbons starts with radiative association of $\mathrm{C}^{+}$and $\mathrm{H}_{2}$, leading to excited $\mathrm{CH}_{2}^{+}$(Herbst 1985).

Ionized by FUV, X-ray photons or CRPs, ions and molecule drive rapid ion-neutral chemistry, which constitutes the largest fraction of astrochemical models. These reactions are exothermic, with high rate coefficients $\sim 10^{-9} \mathrm{~cm}^{-3} \mathrm{~s}^{-1}$, which often increase toward low temperatures $(\beta<0)$ (e.g., Dalgarno \& Black 1976). Ion-neutral reactions result in bond restructuring of the reactants. Some of the most important reactions of this category are protonation reactions.

Molecular ions are destroyed by dissociative recombination with electrons and negatively charged grains. These processes are especially fast at low temperatures, with typical rates of about $10^{-7} \mathrm{~cm}^{-3} \mathrm{~s}^{-1}$ at $10 \mathrm{~K}$ (Woodall et al. 2007). For nearly all observed species, dissociative recombination is an important formation pathway (e.g., water and hydrocarbons). Often, at later evolutionary times, $\gtrsim 10^{5}$ years, dissociative recombination is balanced by protonation reactions, e.g. $\mathrm{CO}+\mathrm{H}_{3}^{+} \rightarrow \mathrm{HCO}^{+}+\mathrm{H}_{2}$ followed by $\mathrm{HCO}^{+}+\mathrm{e}^{-} \rightarrow \mathrm{CO}+\mathrm{H}$. Products and branching ratios for polyatomic ions are not easily obtainable (e.g., Bates \& Herbst 1988; Spanel \& Smith 1994).

A number of neutral-neutral reactions involving open-shell radicals can also be active in both cold outer and warm inner disk regions (van Dishoeck 1998). The typical rate coefficient for these reactions is $\sim 10^{-11}-10^{-10} \mathrm{~cm}^{-3} \mathrm{~s}^{-1}$, i.e., only about an order of magnitude lower than for the ion-molecule processes (e.g., Clary et al. 1994; Smith et al. 2004). One of the most interesting reactions of this type is formation of $\mathrm{HCO}^{+}$upon collision between $\mathrm{O}$ and $\mathrm{CH}\left(\alpha_{0}=2.0 \cdot 10^{-11} \mathrm{~cm}^{-3} \mathrm{~s}^{-1}\right.$; Woodall et al. 2007).

\section{Photochemistry}

The photochemical reactions are overviewed in this book in the paper by van Dishoeck \& Visser and only briefly discussed here.

Young $\mathrm{T}$ Tau stars $\left(T_{\text {eff }} \simeq 4000 \mathrm{~K}\right)$ emit intense non-thermal UV radiation that has a spectra different from the interstellar UV field (Bergin et al. 2003a), with a prominent Lyman $_{\alpha}$ line, while hot $\left(T_{\text {eff }} \gtrsim 10000 \mathrm{~K}\right)$ Herbig Ae/Be stars produce a lot of thermal UV emission. The overall intensity of the stellar UV radiation at $100 \mathrm{AU}$ from the star can be as high as 500 and $10^{5}$ for a T Tau and a Herbig Ae star, respectively, in units of the interstellar UV field (Habing 1968).

Many molecules, such as $\mathrm{CO}, \mathrm{H}_{2}$, and $\mathrm{CN}$, are dissociated by radiation at short wavelengths $(\lambda \lesssim 1100 \AA)$, while photodissociation of other species, such as HCN, occurs at longer wavelengths (van Dishoeck \& Black 1988; van Dishoeck et al. 2006). Some molecules (e.g., $\mathrm{H}_{2}$ and $\mathrm{CO}$ ) dissociate via absorption of UV photons at discrete lines to the excited (Rydberg) states, whereas other molecules are dissociated either by the continuum (e.g., $\mathrm{CH}_{4}$ ) or by the continuum and lines (e.g., $\mathrm{C}_{2}$ ) (van Dishoeck 1988; van Dishoeck et al. 2006). The high ratio of CN to HCN abundances, observed in disks (Dutrey et al. 1997), can be explained by more intense photodissociation of HCN if part of the UV flux comes as $\mathrm{Ly}_{\alpha}$ photons (1216 $\AA$; Bergin et al. 2003a).

Since the dissociation of the abundant $\mathrm{H}_{2}$ and $\mathrm{CO}$ molecules result from photoabsorption at discrete wavelengths, isotopically selective photodissociation based on selfshielding is possible (e.g., Visser et al. 2009a). Two conditions for that are required: 1) dissociation via line absorption for each isotopically substituted molecule, and 2) differential photolysis that depends upon the isotopic abundances. Self-shielding occurs when the spectral lines leading to dissociation of the major isotopic species optically saturate, 
while the other residual lines relevant for dissociation of the minor isotopes remain transparent. Under conditions of protoplanetary disks, isotopic-selective chemistry may occur for abundant CO molecules (e.g., Dalgarno \& Stephens 1970; Thiemens \& Heidenreich 1983; van Dishoeck \& Black 1988; Lee et al. 1996; Visser et al. 2009a).

\section{Gas-grain interactions}

In cold disk regions grains serve as a passive sink for heavy molecules and electrons, while providing free electrons to dissociate positive ions. In the darkest midplane zone dust grains become the dominant charged species (Semenov et al. 2004). At low temperatures of $\lesssim 20-50 \mathrm{~K}$, many molecules adhere to a grain with a nearly $100 \%$ sticking probability since their kinetic energy is much smaller than the binding energy (see, e.g., d'Hendecourt et al. 1985; Buch \& Zhang 1991). This sticking probability also depends on surface properties, such as porosity and distribution of surface chemi- and physisorption sites. Chemisorption requires formation of a chemical bond between a surface species and a grain, and such a species does not evaporate easily (e.g., Cazaux et al. 2005).

The most effective desorption processes for disk chemistry are thermal evaporation, cosmic ray induced desorption, and photodesorption. Thermal evaporation occurs if a molecule has energy that exceeds it's binding energy. Typical binding energies of physisorbed species are about $1000 \mathrm{~K}$ for light molecules like $\mathrm{CO}$ and $\mathrm{N}_{2}$ (Bisschop et al. 2006), and much larger for heavier cyanopolyynes and carbon chains. Chemisorbed species do not desorb until very high temperatures of $100-1000 \mathrm{~K}$ are reached.

In disk midplane CRPs provide energy that allows dust mantles (partly) thermally evaporate. A relativistic iron nucleus may eventually collide with a grain and impulsively heat it, releasing a portion of the volatile component (e.g., Watson \& Salpeter 1972; Leger et al. 1985; Hartquist \& Williams 1990). In less opaque disk regions, penetrating UV photons lead to photoevaporation of surface species. The probability of evaporation per one UV photon has been measured in the laboratory for some simple molecules, such as $\mathrm{CO}, \mathrm{H}_{2} \mathrm{O}, \mathrm{CH}_{4}$, and $\mathrm{NH}_{3}$, and is about $10^{-6}-10^{-2}$ (e.g., Öberg et al. 2007, 2009b,a). The dilute UV radiation field produced by cosmic rays in the disk midplane (Prasad \& Tarafdar 1983) can also be important for high values of the photodesorption rates. Another mechanism is the X-ray induced desorption which is efficient only for tiny grains, $\lesssim 0.05 \mu \mathrm{m}$ (Najita et al. 2001).

\section{Surface formation of complex species}

The surfaces of dust grains serve as a catalyst for reactions that do not proceed efficiently in the gas phase. The most notable example is formation of molecular hydrogen, which occurs almost entirely on dust surfaces (e.g., Hollenbach \& Salpeter 1971; Watson \& Salpeter 1972). Tielens \& Hagen (1982) have studied the chemical evolution of surface species on large grains and considered the effect of various migration rates for light atoms and heavy molecules. A typical $0.1 \mu \mathrm{m}$ amorphous silicate grain accommodates $\sim 10^{6}$ surface sites available for accretion. An atom or light radical, if it is not chemisorbed, may migrate over the surface from site to site by thermal hopping, when its energy exceeds the barriers for particle motions and react with other species.

There is an increasing body of evidence from laboratory measurements that complex molecules like methanol cannot be produced in the gas phase via radiative association forming a large protonated precursor followed by its dissociative recombination (Geppert et al. 2005). Thus, surface reactions due to thermal hopping remain the only viable 
formation pathway for production of complex (organic) molecules in protoplanetary disks, found also in meteoritic samples in Solar system.

\section{Importance of dynamical processes for disk chemistry}

While most of the chemical studies are still based on laminar disk models, evidences for mixing call for a more sophisticated treatment. Models of the early Solar nebula with radial transport by advective flows have been developed (e.g., Morfill \& Völk 1984; Gail 2001, 2002; Wehrstedt \& Gail 2002; Boss 2004; Keller \& Gail 2004). Ilgner et al. (2004) for the first time modeled the influence of turbulent diffusion in the vertical direction and advection flows in the radial direction on the chemical composition of the inner disk region. They found that dynamical processes significantly affect the chemical evolution of sulfur-bearing species. Willacy et al. (2006) have shown that 1D vertical mixing modifies chemical composition of the outer disk region and that the mixing results better agree to observations. Semenov et al. (2006, Paper I) and Aikawa (2007) have found that turbulent transport allows explaining the presence of a large amount of cold $(\lesssim 15 \mathrm{~K})$ CO gas in the disk of DM Tau. Tscharnuter \& Gail (2007) have used a 2D disk chemo-hydrodynamical model and showed that in the disk midplane matter moves outward, carrying out the angular momentum, while the accretion flows toward the star are located at elevated altitudes. Consequently, gas-phase species produced by warm chemistry in the inner nebula can be steadily transported into the cold outer region and freeze out. A radial advection model has also been utilized by Nomura et al. (2009), who have demonstrated that inward radial transport enhances abundances of organic molecules (produced mainly on dust surfaces in cold outer regions). Hersant et al. (2009) have studied various mechanisms to retain gas-phase $\mathrm{CO}$ in very cold disk regions. They concluded that efficient photodesorption in moderately obscured disk regions $\left(A_{\mathrm{V}}<5^{\mathrm{m}}\right)$ greatly enhances gas-phase CO concentrations, while the role of vertical mixing is less important. Finally, Heinzeller et al. (2011) have investigated the disk chemical evolution with radial advection, vertical mixing, and vertical wind transport processes. They have found that the disk wind has a negligible effect on disk chemistry, whereas the radial accretion alters the molecular abundances in the cold midplane, and the vertical turbulent mixing affects the chemistry in the warm molecular layer.

We study the influence of turbulence mixing on the chemical evolution of protoplanetary disks in Semenov \& Wiebe (2011). Mixing is important in disks since a chemical steady-state is not reached due to long timescales associated with surface chemical processes and slow evaporation of heavy molecules. Our analysis was based on the $\alpha$-model of a $\sim 5$ Myr DM Tau disk coupled to the large-scale gas-grain chemical code "ALCHEMIC" (Semenov et al. 2010b). To account for production of complex molecules, our chemical network was supplied with a set of surface reactions and photoprocessing of ices.

The adopted flaring disk structure is based on a $1+1 \mathrm{D}$ steady-state $\alpha$-model similar to D'Alessio et al. (1999) model. The non-thermal FUV radiation field from DM Tau is represented by the scaled ISRF of Draine (1978), with the un-attenuated intensity at $100 \mathrm{AU}$ of $\chi_{*}(100)=410$ (e.g., Bergin et al. 2003b). For the X-ray luminosity of the star we adopt a value of $10^{30} \mathrm{erg} \mathrm{s}^{-1}$, which is constrained by recent measurements with Chandra and XMM in the range of $0.3-10 \mathrm{keV}$.

The turbulence in disks is likely driven by the magnetorotational instability (MRI), which is operative even in a weakly ionized medium (e.g., Balbus \& Hawley 1991). This turbulence causes anomalous viscosity that enables efficient redistribution of the angular momentum. We have followed the parametrization of Shakura \& Sunyaev (1973), where 
Table 3. Detectable tracers of turbulent mixing.

\begin{tabular}{ll}
\hline Steadfast & Hypersensitive \\
\hline $\mathrm{CO}$ & Heavy hydrocarbons (e.g., $\left.\mathrm{C}_{6} \mathrm{H}_{6}\right)$ \\
$\mathrm{H}_{2} \mathrm{O}$ ice & $\mathrm{C}_{2} \mathrm{~S}$ \\
& $\mathrm{C}_{3} \mathrm{~S}$ \\
& $\mathrm{CO}_{2}$ \\
& $\mathrm{O}_{2}$ \\
& $\mathrm{SO}$ \\
& $\mathrm{SO}_{2}$ \\
& $\mathrm{OCN}$ \\
& Complex organics (e.g., $\mathrm{HCOOH})$ \\
\hline
\end{tabular}

turbulent viscosity $\nu$ is related to local disk properties such as the vertical spatial scale $H(r)$, the sound speed $c_{\mathrm{s}}(r, z)$, and the dimensionless parameter $\alpha$ :

$$
\nu(r, z)=\alpha c_{\mathrm{S}}(r, z) H(r) .
$$

From observational constraints $\alpha$ is $\sim 0.001-0.1$ (Andrews \& Williams 2007; Guilloteau et al. 2011), so we adopt the constant value of 0.01. Consequently, the diffusion coefficient is calculated as

$$
D_{\text {turb }}(r, z)=\nu(r, z) / S c
$$

where $S c$ is the Schmidt number describing efficiency of turbulent diffusivity (see e.g. Shakura \& Sunyaev 1973; Schräpler \& Henning 2004). We assume that gas-phase species and dust grains are well mixed, and transported with the same diffusion coefficient.

We show that the higher the ratio of the characteristic chemical timescale to the turbulent transport timescale for a given molecule, the higher the probability that its column density will be affected by dynamical processes. Thus, turbulent transport influences abundances of many gas-phase species and especially ices. Vertical mixing is more important as it affects the evolution of gas-phase and surface species of any kind, whereas the effect of radial mixing is pronounced mostly for the evolution of ices. The radial temperature gradient is weaker, and thus is only relevant for the evolution of polyatomic ices formed via surface reactions of heavy radicals, whereas steep vertical gradients of temperature and high-energy radiation intensity cause much sharper transition from the ice-dominated chemistry in the disk midplane to the rich gas-phase chemistry in the molecular layer.

The simple molecules that are unresponsive to dynamical transport include $\mathrm{C}_{2} \mathrm{H}, \mathrm{C}^{+}$, $\mathrm{CH}_{4}, \mathrm{CN}, \mathrm{CO}, \mathrm{HCN}, \mathrm{HNC}, \mathrm{H}_{2} \mathrm{CO}, \mathrm{OH}$, as well as water and ammonia ices. The sensitive species to dynamics are carbon chains and other heavy species, in particular sulfurbearing and complex organic molecules frozen onto the dust grains. Mixing steadily transports ice-coated grains in warmer regions, allowing more efficient surface processing due to enhanced hopping rates of heavy radicals. In warm intermediate layer these organically-rich ices evaporate, and in the inner disk they can also be photodissociated by CRP/X-ray-induced UV photons. The importance of mixing is higher in an inner, planet-forming disk zone, where thermal, density, and high-energy radiation gradients are stronger than in the outer region. Still, mixing does not completely erase the layered chemical structure of protoplanetary disks.

Several promising detectable tracers of dynamical processes in protoplanetary disks are the column density ratios of the $\mathrm{CO}_{2}, \mathrm{O}_{2}$, SO, $\mathrm{SO}_{2}, \mathrm{C}_{2} \mathrm{~S}, \mathrm{C}_{3} \mathrm{~S}$ to that of $\mathrm{CO}$ and the water ice (see Table 3 ). The detection of complex species (e.g., dimethyl ether, formic acid, 
methyl formate, etc.) in protoplanetary disks with ALMA and JWST will be a strong indication that chemical evolution of these objects is influenced by transport processes.

\section{References}

Agúndez, M., Cernicharo, J., \& Goicoechea, J. R. 2008, A\&A, 483, 831

Aikawa, Y. 2007, ApJ, 656, L93

Aikawa, Y. \& Herbst, E. 1999, A\&\&A, 351, 233

Aikawa, Y., Momose, M., Thi, W.-F., et al. 2003, PASJ, 55, 11

Aikawa, Y., Umebayashi, T., Nakano, T., \& Miyama, S. M. 1999, ApJ, 519, 705

Andrews, S. M. \& Williams, J. P. 2007, ApJ, 659, 705

Balbus, S. A. \& Hawley, J. F. 1991, ApJ, 376, 214

Bates, D. R. 1951, MNRAS, 111, 303

Bates, D. R. \& Herbst, E. 1988, in ASSL Vol. 146: Rate Coefficients in Astrochemistry, ed. T. J. Millar \& D. A. Williams (Kluwer Academic Publishers, Dordrecht), 41-48

Bergin, E., Calvet, N., D'Alessio, P., \& Herczeg, G. J. 2003a, ApJ, 591, L159

Bergin, E., Calvet, N., D'Alessio, P., \& Herczeg, G. J. 2003b, ApJ, 591, L159

Bergin, E. A., Aikawa, Y., Blake, G. A., \& van Dishoeck, E. F. 2007, in Protostars and Planets V, ed. B. Reipurth, D. Jewitt, \& K. Keil, 751-766

Bisschop, S. E., Fraser, H. J., Öberg, K. I., van Dishoeck, E. F., \& Schlemmer, S. 2006, A\&্A, 449,1297

Boss, A. P. 2004, ApJ, 616, 1265

Bouvier, J., Alencar, S. H. P., Harries, T. J., Johns-Krull, C. M., \& Romanova, M. M. 2007, Protostars and Planets V, 479

Bouwman, J., Henning, T., Hillenbrand, L. A., et al. 2008, ApJ, 683, 479

Bradley, J. P. 2005, in Meteorites, Comets and Planets: Treatise on Geochemistry, Volume 1, ed. A. M. Davis, H. D. Holland, \& K. K. Turekian (Elsevier B), 689-+

Brownlee, D. E., Horz, F., Newburn, R. L., et al. 2004, Science, 304, 1764

Brownlee, D. E., Joswiak, D. J., Matrajt, G., Bradley, J. P., \& Ebel, D. S. 2008, in Lunar and Planetary Institute Conference Abstracts, Vol. 39, Lunar and Planetary Institute Conference Abstracts, 1978-+

Buch, V. \& Zhang, Q. 1991, ApJ, 379, 647

Carr, J. S. \& Najita, J. R. 2008, Science, 319, 1504

Cazaux, S., Caselli, P., Tielens, A. G. G. M., LeBourlot, J., \& Walmsley, M. 2005, Journal of Physics Conference Series, 6, 155

Ciesla, F. J. 2009, Icarus, 200, 655

Clary, D. C., Haider, N., Husain, D., \& Kabir, M. 1994, ApJ, 422, 416

D'Alessio, P., Calvet, N., Hartmann, L., Lizano, S., \& Cantó, J. 1999, ApJ, 527, 893

Dalgarno, A. \& Black, J. H. 1976, Reports of Progress in Physics, 39, 573

Dalgarno, A. \& Stephens, T. L. 1970, ApJ, 160, L107

Dartois, E., Dutrey, A., \& Guilloteau, S. 2003, A\&SA, 399, 773

d'Hendecourt, L. B., Allamandola, L. J., \& Greenberg, J. M. 1985, A\&\&A, 152, 130

Draine, B. T. 1978, ApJS, 36, 595

Dutrey, A., Guilloteau, S., \& Guelin, M. 1997, A\&A, 317, L55

Dutrey, A., Guilloteau, S., \& Ho, P. 2007a, in Protostars and Planets V, ed. B. Reipurth, D. Jewitt, \& K. Keil, 495-506

Dutrey, A., Guilloteau, S., Piétu, V., et al. 2008, A\&\&A, 490, L15

Dutrey, A., Henning, T., Guilloteau, S., et al. 2007b, A\&A, 464, 615

Flynn, G. J., Bleuet, P., Borg, J., et al. 2006, Science, 314, 1731

Gail, H.-P. 2001, $A \& A$ A, 378, 192

Gail, H.-P. 2002, A\&BA, 390, 253

Geppert, W. D., Hellberg, F., Österdahl, F., et al. 2005, in IAU Symposium, Vol. 231, Astrochemistry: Recent Successes and Current Challenges, ed. D. C. Lis, G. A. Blake, \& E. Herbst, 117-124 
Glassgold, A. E., Najita, J., \& Igea, J. 1997, ApJ, 480, 344

Güdel, M. \& Nazé, Y. 2009, A\&\&A Rev., 17, 309

Guilloteau, S., Dutrey, A., Piétu, V., \& Boehler, Y. 2011, A\&A, 529, A105+

Habart, E., Natta, A., \& Krügel, E. 2004, A\&SA, 427, 179

Habing, H. J. 1968, Bull. Astron. Inst. Netherlands, 19, 421

Hartquist, T. W. \& Williams, D. A. 1990, MNRAS, 247, 343

Heinzeller, D., Nomura, H., Walsh, C., \& Millar, T. J. 2011, ApJ, 731, 115

Henning, T., Semenov, D., Guilloteau, S., et al. 2010, ApJ, 714, 1511

Herbst, E. 1985, ApJ, 291, 226

Herbst, E. \& Klemperer, W. 1973, ApJ, 185, 505

Hersant, F., Wakelam, V., Dutrey, A., Guilloteau, S., \& Herbst, E. 2009, A\&3A, 493, L49

Hollenbach, D. \& Salpeter, E. E. 1971, ApJ, 163, 155

Hughes, A. M., Andrews, S. M., Espaillat, C., et al. 2009, ApJ, 698, 131

Hughes, A. M., Wilner, D. J., Andrews, S. M., Qi, C., \& Hogerheijde, M. R. 2011, ApJ, 727, 85

Ilgner, M., Henning, T., Markwick, A. J., \& Millar, T. J. 2004, A\&A A, 415, 643

Isella, A., Natta, A., Wilner, D., Carpenter, J. M., \& Testi, L. 2010, ApJ, 725, 1735

Juhász, A., Bouwman, J., Henning, T., et al. 2010, ApJ, 721, 431

Kastner, J. H., Zuckerman, B., Weintraub, D. A., \& Forveille, T. 1997, Science, 277, 67

Keller, C. \& Gail, H. 2004, A\&A, 415, 1177

Lahuis, F., van Dishoeck, E. F., Boogert, A. C. A., et al. 2006, ApJ, 636, L145

Le Petit, F., Nehmé, C., Le Bourlot, J., \& Roueff, E. 2006, ApJS, 164, 506

Lee, H.-H., Herbst, E., Pineau des Forets, G., Roueff, E., \& Le Bourlot, J. 1996, A\&SA, 311, 690

Leger, A., Jura, M., \& Omont, A. 1985, A\& $A$, 144, 147

Lin, D. N. C. \& Papaloizou, J. 1980, MNRAS, 191, 37

Lissauer, J. J. 1987, Icarus, 69, 249

Markwick, A. J., Ilgner, M., Millar, T. J., \& Henning, T. 2002, A $\& A$ A, 385, 632

Morfill, G. E. \& Völk, H. J. 1984, ApJ, 287, 371

Najita, J., Bergin, E. A., \& Ullom, J. N. 2001, ApJ, 561, 880

Nomura, H., Aikawa, Y., Nakagawa, Y., \& Millar, T. J. 2009, A\&\&A, 495, 183

Öberg, K. I., Fuchs, G. W., Awad, Z., et al. 2007, ApJ, 662, L23

Öberg, K. I., Linnartz, H., Visser, R., \& van Dishoeck, E. F. 2009a, ApJ, 693, 1209

Öberg, K. I., van Dishoeck, E. F., \& Linnartz, H. 2009b, A\&\&A, 496, 281

Panić, O., Hogerheijde, M. R., Wilner, D., \& Qi, C. 2009, A\&̋A, 501, 269

Pascucci, I., Apai, D., Luhman, K., et al. 2009, ApJ, 696, 143

Piétu, V., Dutrey, A., \& Guilloteau, S. 2007, A\&A, 467, 163

Piétu, V., Guilloteau, S., \& Dutrey, A. 2005, A\&A, 443, 945

Pontoppidan, K. M., Blake, G. A., van Dishoeck, E. F., et al. 2008, ApJ, 684, 1323

Pontoppidan, K. M., Dullemond, C. P., van Dishoeck, E. F., et al. 2005, ApJ, 622, 463

Prasad, S. S. \& Tarafdar, S. P. 1983, ApJ, 267, 603

Qi, C., Kessler, J. E., Koerner, D. W., Sargent, A. I., \& Blake, G. A. 2003, ApJ, 597, 986

Qi, C., Wilner, D. J., Aikawa, Y., Blake, G. A., \& Hogerheijde, M. R. 2008, ApJ, 681, 1396

Qi, C., Wilner, D. J., Calvet, N., et al. 2006, ApJ, 636, L157

Salyk, C., Pontoppidan, K. M., Blake, G. A., et al. 2008, ApJ, 676, L49

Salyk, C., Pontoppidan, K. M., Blake, G. A., Najita, J. R., \& Carr, J. S. 2011, ApJ, 731, 130

Schräpler, R. \& Henning, T. 2004, ApJ, 614, 960

Semenov, D., Chakraborty, S., \& Thiemens, M. 2010a, Chemical and Isotopic Evolution of the

Solar Nebula and Protoplanetary Disks, ed. Apai, D. A. \& Lauretta, D. S., 97-127

Semenov, D., Hersant, F., Wakelam, V., et al. 2010b, A\&A A, 522, A42+

Semenov, D. \& Wiebe, D. 2011, ArXiv e-prints, 1104.4358

Semenov, D., Wiebe, D., \& Henning, T. 2004, A\&A, 417, 93

Semenov, D., Wiebe, D., \& Henning, T. 2006, ApJ, 647, L57

Shakura, N. I. \& Sunyaev, R. A. 1973, $A \& A$, 24, 337

Smith, I. W. M., Herbst, E., \& Chang, Q. 2004, MNRAS, 350, 323

Spanel, P. \& Smith, D. 1994, Chemical Physics Letters, 229, 262 
Terada, H., Tokunaga, A. T., Kobayashi, N., et al. 2007, ApJ, 667, 303

Thi, W.-F., van Zadelhoff, G.-J., \& van Dishoeck, E. F. 2004, A\&SA, 425, 955

Thiemens, M. H. \& Heidenreich, III, J. E. 1983, Science, 219, 1073

Tielens, A. G. G. M. \& Hagen, W. 1982, A\&AA, 114, 245

Tscharnuter, W. M. \& Gail, H.-P. 2007, A\&A, 463, 369

van Boekel, R., Min, M., Leinert, C., et al. 2004, Nature, 432, 479

van den Ancker, M. E., Bouwman, J., Wesselius, P. R., et al. 2000, A\&\&A, 357, 325

van der Plas, G., van den Ancker, M. E., Acke, B., et al. 2009, A\& A, 500, 1137

van Dishoeck, E. F. 1988, in ASSL Vol. 146: Rate Coefficients in Astrochemistry, ed. T. Millar \& D. Williams (Kluwer Academic Publishers, Dordrecht), 49-72

van Dishoeck, E. F. 1998, in The Molecular Astrophysics of Stars and Galaxies, ed. T. W. Hartquist \& D. A. Williams (Clarendon Press, Oxford), 53-100

van Dishoeck, E. F. 2004, ARA\&A, 42, 119

van Dishoeck, E. F. \& Black, J. H. 1988, ApJ, 334, 771

van Dishoeck, E. F., Jonkheid, B., \& van Hemert, M. C. 2006, in Faraday discussion, Vol. 133, Chemical evolution of the Universe, ed. I. R. Sims \& D. A. Williams, 231-244

van Zadelhoff, G.-J., Aikawa, Y., Hogerheijde, M. R., \& van Dishoeck, E. F. 2003, A\&A, 397, 789

Visser, R., van Dishoeck, E. F., \& Black, J. H. 2009a, A\&A, 503, 323

Visser, R., van Dishoeck, E. F., Doty, S. D., \& Dullemond, C. P. 2009b, A\&\&A, 495, 881

Wakelam, V. 2009, in American Astronomical Society Meeting Abstracts, Vol. 214, American Astronomical Society Meeting Abstracts, 402.15-+

Wakelam, V., Smith, I. W. M., Herbst, E., et al. 2010, Space Sci. Rev., 156, 13

Walsh, C., Millar, T. J., \& Nomura, H. 2010, ArXiv e-prints

Watson, W. D. \& Salpeter, E. E. 1972, ApJ, 174, 321

Wehrstedt, M. \& Gail, H. 2002, A\&SA, 385, 181

Willacy, K., Langer, W., Allen, M., \& Bryden, G. 2006, ApJ, 644, 1202

Williams, D. A. 1972, Astrophys. Lett., 10, L17

Woodall, J., Agúndez, M., Markwick-Kemper, A. J., \& Millar, T. J. 2007, A $\& A, ~ 466,1197$

Woods, P. M. \& Willacy, K. 2009, ApJ, 693, 1360

Zasowski, G., Kemper, F., Watson, D. M., et al. 2009, ApJ, 694, 459

\section{Discussion}

PAul M. Woods: There were some dynamical models recently that show that the accretion flow proceeds inwards along the disc surface and outwards along the disc midplane. Are those models credible, and how would that affect the disc chemistry?

D. Semenov: Turbulence is a $3 \mathrm{D}$ phenomenon and its magnitude varies greatly across a disk. Global 3D MHD models show that viscous transport occurs in all directions in any given disk location, albeit with different efficiencies, and that there is no a specific direction for it. According to observations, advective transport seems to be an effective mechanism that regulates overall lifetime of disks, $\sim 1-10 \mathrm{Myr}$, in which most of disk matter accretes onto the central star and photoevaporates. Consequently, disk structure changes with time, as well as the FUV and X-ray penetration, and grain properties. This strongly affects chemical evolution of protoplanetary disks, and favors the use of evolutionary disk models (see R. Vissier's presentation and proceeding paper.

JAMES R. LyONS: What turbulent viscosity parameter $\alpha$-values were used for slow vs. fast mixing?

D. Semenov: We considered Fickian diffusion, in which the diffusion coefficient $D_{\text {turb }}$ $(r, z)$ is attributed to the $\alpha$-parameterized viscosity, $\alpha=0.01: D_{\text {turb }}(r, z)=\nu(r, z) / S c=$ $\alpha c_{\mathrm{s}}(r, z) H(r) / S c$, where the characteristic spatial scale is $H(r)$ and the sound speed is 
$c_{\mathrm{S}}(r, z)$. The $S c$ the Schmidt number describing efficiency of turbulent diffusivity. The "fast" mixing model has $S c=1$ and the "slow" model has "Sc=100".

JAMES R. LyONS: Have you included isotopes in vertical mixing calculations?

D. Semenov: No.

JAmes R. Lyons: What did you mean by "isotopic homogeneity of the Solar nebula"? It is not homogeneous in oxygen isotopes.

D. Semenov: I've meant that the inner, 1-20 AU region of the Solar nebula has been isotopically homogenized at bulk level, though oxygen anomalies at percentage level still persist. 